\title{
A CLASS OF HYPO-DIRICHLET ALGEBRAS
}

\author{
A. G. BRANDSTEIN
}

ABstract. A method is given of constructing a new class of hypo-Dirichlet algebras of given real codimension.

1. Introduction. Let $X$ be a compact Hausdorff space and $A$ a uniform algebra on $X$, i.e., a uniformly closed subalgebra of $C(X)$, the space of continuous functions on $X$, that contains the constants and separates points on $X$. Denote the real parts of the functions in $A$ by $\operatorname{Re} A$, the set of invertible elements of $A$ by $A^{-1}$, the set of logarithms of moduli of functions in $A$ by $\log A$. Let $C(X)$ denote the space of real continuous functions on $X$. A uniform algebra on $X$ is called a hypoDirichlet algebra if, in addition, there exist $f_{1}, \cdots, f_{n}$ in $A^{-1}$, such that the (real) vector space spanned by $\operatorname{Re} A$ and $\log \left|f_{1}\right|, \ldots$, $\log \left|f_{n}\right|$ is dense in $C(X)$. The minimal number of such ' $f_{i}$ ' required shall be called the codimension of $\operatorname{Re} A$. Hypo-Dirichlet algebras were first studied by Wermer [6], and further investigated by Ahern and Sarason [1]. The object here is to exhibit a class of examples of such algebras. The proofs of several of the lemmas in this paper are modeled after [2].

2. The algebra $A$. Let $\Gamma$ be the annulus $\{Z: 1 \leqq|Z| \leqq 2\}, \gamma_{1}$ $=\{Z:|Z|=1\}$ and $\gamma_{2}=\{Z:|Z|=2\}$. Let $\Psi$ be a homeomorphism of $\gamma_{1}$ on $\gamma_{2}$ which is orientation-preserving and singular, i.e., maps a Borel set of one-dimensional Lebesgue measure 0 onto a set of measure $4 \pi$. Let $B=\{f \in C(\Gamma): f$ is analytic in int $(\Gamma)\}$, and $A$ $=\{f \in B: f(Z)=f(\Psi(Z))\}$ for all $Z \in \gamma_{1}$. Let $A_{\Psi}=A$ restricted to $\gamma_{1}$. Then $A_{\Psi}$ is a uniformly closed algebra of continuous functions on $\gamma_{1}$, which contains the constants.

Theorem. $A_{\Psi}$ is a hypo-Dirichlet algebra on $\gamma_{1}$, and $\operatorname{Re} A_{\Psi}$ has codimension 1 in $C_{R}\left(\gamma_{1}\right)$.

Definition 1. A (complex Borel) measure $\nu$ on $\gamma_{1} \cup \gamma_{2}$ is odd if for each Borel set $E \subset \gamma_{1}, \nu(E)=-\nu(\Psi(E))$.

Definition 2. $H$ denotes the class of measures of the form: $g(Z) d Z$ on $\gamma_{1} \cup \gamma_{2}$, where $g$ is any function in the $L^{1}$ closure of $B$ restricted to $\gamma_{1} \cup \gamma_{2}$.

Received by the editors July 23, 1969 .

AMS 1970 subject classifications. Primary 46J10, 46J20.

Key words and phrases. Real condimension, singular homeomorphism, cohomology.

Copyright (c) 1971, American Mathematical Society 
Definition 3. $W$ is the space of measures $\mu+\nu$ with $\mu \in H, \nu$ odd. $\bar{W}$ is the weak * closure of $W$ in the space of measures on $\gamma_{1} \cup_{\gamma_{2}}$.

Definition 4. A measure $\lambda$ on $\gamma_{1} \cup \gamma_{2}$ annihilates $A$ if $\int f d \lambda=0$, all $f \in A$.

Clearly, every measure in $W$ annihilates $A$. Also, if $\lambda$ annihilates $A$, then $\lambda \in \bar{W}$.

Note. The measure $-i \cdot(d Z / Z)=d \theta$ is a real measure which annihilates $B$, and it is readily seen that the only real annihilators of $B$ are of the form: $\alpha \cdot d \theta, \alpha$ a real constant.

LEммA 1. If $\mu \in H, \nu$ odd, then $\|\nu\| \leqq 16\|\mu+\nu\|$.

Proof. Let $E$ be any Borel subset of $\gamma_{1}$ and let $m$ represent Lebesgue measure. Then there are disjoint sets $F$ and $G$ with $E=F \cup G$, $m(F)=m(\Psi(G))=0$. Let $K=\|\mu+\nu\|$, then $|\nu(F)|=|\nu(F)+\mu(F)| \leqq K$, since $\mu$ is absolutely continuous. $|\nu(G)|=|\nu(\Psi(G))|=|(\mu+\nu)(\Psi(G))|$ $\leqq K$ for the same reason. Hence $\|\nu\| \leqq 16 K$. q.e.d.

Lemma 2. Then $W=\bar{W}$.

Proof. $Q=\{\mu+\nu: \mu \in H, \nu$ odd, $\|\mu\| \leqq 1,\|\nu\| \leqq 1\}$ is compact. The Krein-Smulian theorem [4, p. 429] then implies $W=\bar{W}$. q.e.d.

LEMмA 3. If $\nu$ is an odd measure, then $\nu$ is absolutely continuous with respect to arc length on $\gamma_{1} \cup \gamma_{2}$ iff $\nu=0$.

Proof. Suppose $\nu$ is absolutely continuous. Let $E$ be a Borel subset of $\gamma_{1}$. Then there are disjoint sets $F$ and $G$ with $E=F \cup G, m(F)$ $=m(\Psi(G))=0$. Hence $\nu(F)=0$, since $\nu$ is absolutely continuous $\nu(G)=-\nu(\Psi(G))=0$ for the same reason. Hence $\nu(E)=0$. q.e.d.

Lemma 4. Every real annihilator, $\lambda$, of $A$ has the form: $\lambda=\nu+\alpha \cdot d \theta$, where $\nu$ is odd and $\alpha$ is a real scalar.

Proof. Since $W$ is weak * closed, we conclude that if $\lambda$ is a measure on $\gamma_{1} \cup \gamma_{2}$, which annihilates $A$, then $\lambda=\mu+\nu, \mu \in H, \nu$ odd. Write $\mu=\mu_{1}+i \mu_{2}, \nu=\nu_{1}+i \nu_{2}$ with $\mu_{1}, \mu_{2}, \nu_{1}$, and $\nu_{2}$ real. If $\lambda$ is real $\mu_{2}+\nu_{2}=0$. Hence $\nu_{2}$ is absolutely continuous, hence 0 . Then $\mu=\mu_{1}$ and $\lambda=\mu_{1}+\nu_{1}$. q.e.d.

It follows, in particular, that $\operatorname{Re} A$ has codimension $\leqq 1$ in $C_{R}\left(\gamma_{1}\right)$.

Lemma 5. A separates the points of $\gamma_{1}$. Further, given $Z_{1}, Z_{2}$ with $1 \leqq\left|Z_{1}\right| \leqq 2,1 \leqq\left|Z_{2}\right|<2$ and $Z_{1} \neq Z_{2}$, then there exists an $f$ in $A$ such that $f\left(Z_{1}\right) \neq f\left(Z_{2}\right)$.

Proof. Let $\tau_{1}, \tau_{2}$ be two points of $\gamma_{1}$ and let $\delta_{\tau_{1}}, \delta_{\tau_{2}}$ be the point masses at $\tau_{1}, \tau_{2}$ respectively. Unless $A$ separates $\tau_{1}$ and $\tau_{2}, \delta_{\tau_{1}}-\delta_{\tau_{2}}$ will 
be a real annihilating measure which is not in $W$. Now suppose $Z_{1}, Z_{2}$ are interior to the annulus and $A$ fails to separate them. Let $\sigma_{1}, \sigma_{2}$ be the harmonic measures for $Z_{1}, Z_{2}$ respectively. Then $\sigma_{1}-\sigma_{2}$ would be a real annihilating measure. Hence, $\sigma_{1}-\sigma_{2}=\nu+\alpha \cdot d \theta, \nu$ odd. However $\sigma_{1}-\sigma_{2}$ is absolutely continuous, therefore $\sigma_{1}-\sigma_{2}=\alpha \cdot d \theta$, contradiction. Finally, if $Z_{1} \in \gamma_{1}$ and $Z_{2}$ is interior, a similar argument applies. q.e.d.

Let $T$ be the space obtained from the closed annulus $1 \leqq|Z| \leqq 2$ by by identifying $Z$ and $\Psi(Z)$ if $Z \in \gamma_{1}$. Then functions in $A$ may be regarded as continuous functions on $T$. Evidently $T$ is topologically a torus since $\Psi$ is orientation-preserving.

LEMMA 6. The space of maximal ideals of $A\left(A_{\Psi}\right)$ is homeomorphic to $T$.

Proof. It must be shown that, if $h$ is a homomorphism of $A$ onto the complex numbers, then $h$ is evaluation at some point of $T$. If $h$ is not evaluation at any point of $T$, then for each $Z, 1 \leqq|Z| \leqq 2$, there is an $f_{Z} \in A$, with $h\left(f_{z}\right)=0, f_{Z}(Z) \neq 0$. Since $T$ is compact, we can select a finite number of functions $f_{1}, \cdots, f_{n}$ in $A$ such that $h\left(f_{i}\right)=0$ and open sets $\Delta_{i}$ in $1 \leqq|Z| \leqq 2$ such that $U_{1}^{n} \Delta_{i}=\{Z: 1 \leqq|Z| \leqq 2\}$ and $f_{i} \neq 0$ in $\Delta_{i}$. Let $\sigma$ be a representing measure for $h$ on $\gamma_{1} \cup_{\gamma_{2}}$, i.e., $h(f)=\int f d \sigma$, all $f \in A$. Then $\int f \cdot f_{i} d \sigma=h\left(f \cdot f_{i}\right)=h(f) \cdot h\left(f_{i}\right)=0, i=1, \cdots$, $n, f \in A$. Thus $f_{i} \cdot d \sigma$ annihilates $A$, therefore $f_{i} \cdot d \sigma=d \mu_{i}+d \nu_{i}, \mu_{i} \in H$, $\nu_{i}$ odd. Hence, $f_{j}\left(d \mu_{i}+d \nu_{i}\right)=f_{j} \cdot f_{i} d \sigma=f_{i} \cdot\left(d \mu_{j}+d \nu_{j}\right)$ and so $f_{j} \cdot d \mu_{i}$ $-f_{i} d \mu_{j}=f_{i} \cdot d \nu_{j}-f_{j} \cdot d \nu_{i}$. Since the right side is odd and the left side is absolutely continuous both sides vanish. Let $\Phi_{i}$ denote the function in $H$ such that $d \mu_{i}=\Phi_{i} \cdot d Z$. Then $f_{j} \cdot \Phi_{i}=f_{i} \cdot \Phi_{j}$ a.e. on $\gamma_{1} \cup \gamma_{2}$ and so $f_{j} \cdot \Phi_{i}=f_{i} \cdot \Phi_{j}$ also for $1<|Z|<2$. We can therefore unambiguously define $\Phi$ on $1 \leqq|Z| \leqq 2$ by $\Phi(z)=\Phi_{i}(Z) \cdot\left(f_{i}(Z)\right)^{-1}$ for $Z \in \Delta_{i}$. Then $\Phi \in H$.

We define a measure $\nu$ on $\gamma_{1} \cup \gamma_{2}$ by $d \nu=\left(f_{i}\right)^{-1} \cdot d \nu_{i}$ on $\left(\gamma_{1} \cup \gamma_{2}\right) \cap \Delta_{i}$. Then $\nu$ is well defined and odd. Then $f_{i} d \sigma=f_{i} \cdot \Phi \cdot d Z+f_{i} d \nu$. Since $f_{i} \neq 0$ on $\Delta_{i}$, we deduce $d \sigma=\Phi d Z+d \nu$. But then $1=\int d \sigma=\int \Phi \cdot d Z+\int d \nu$ $=0$. Contradiction. q.e.d.

LEмма 7. There is an $f \in A^{-1}$ whose logarithm is not single valued on $\Gamma$.

Proof. We regard $A$ as an algebra of continuous functions on $T$. The circle: $|Z|=3 / 2$ gives rise to a one-cycle $l_{1}$ on $T$. Let $l_{2}$ be another one-cycle on $T$ so that $l_{1}$ and $l_{2}$ generate $H_{1}(T, Z)$. By a theorem of Arens-Royden, [5], the quotient group $A^{-1} / \exp (A)$ is isomorphic to $H^{1}(T, Z)$. If $T$ is the torus, $H^{1}(T, Z)$ is a free abelian group on two 
generators. Let $g_{1}, g_{2}$ be two elements of $A^{-1}$ representing these generators. Write $g_{1}=e^{h_{1}}, g_{2}=e^{h_{2}}$, where $h_{1}, h_{2}$ are (multi-valued) analytic functions on $\Gamma$. Let $h_{1}$ have period $2 n \pi i$ on $l_{2}, h_{2}$ have period $2 m \pi i$ on $l_{2}$. Then $m \cdot h_{1}-n \cdot h_{2}$ has 0 period on $l_{2}$. Suppose it also had 0 period on $l_{1}$. Then $g_{1}^{m} \cdot g^{-n}=e^{h}$ for some $h \in A$. This contradicts the choice of $g_{1}, g_{2}$. Hence $m h-n h$ has period $\neq 0$ on $l$. Therefore $f$ $=g_{1}^{m} \cdot g_{2}^{-n}$ is the desired element of $A^{-1}$. q.e.d.

Proof of TheOREM. We must show that there is an $f \in A_{\psi}^{-1}$ such that $\log |f| \notin$ closure $\operatorname{Re} A$. We claim the $f$ of Lemma 7 is such a function. Define a linear functional $L$ on $C_{R}\left(\gamma_{1} \cup \gamma_{2}\right)$ by $L(U)=$ $(1 / 2 \pi) \int_{|Z|-3 / 2} d v$ where $v$ is the harmonic conjugate of $U$. Then $L$ is continuous and linear. $L(g)=0$ for $g \in \operatorname{Re} A^{-1}$, but $L(\log |f|) \neq 0$ since $\int_{|Z|=3 / 2}(\arg f) \neq 0$. Therefore $\log |f| \notin$ closure $\operatorname{Re} A^{-1}$. q.e.d.

Note. By identifying $n$ circles instead of 2 , in a similar manner, we can construct a hypo-Dirichlet algebra that has real part of codimension $n-1$.

\section{REFERENCES}

1. P. R. Ahern and D. Sarason, The $H^{p}$ spaces of a class of function algebras, Acta Math. 117 (1967), 123-163. MR 36 \#689.

2. A. Browder and J. Wermer, Some algebras of functions on an arc, J. Math. Mech. 12 (1963), 119-130. MR $26 \# 1770$.

3. - A method for constructing Dirichlet algebra, Proc. Amer. Math. Soc. 15 (1964), 546-552. MR 29 \#2669.

4. N. Dunford and J. Schwartz, Linear operators, I: General theory, Pure and Appl. Math., vol. 7, Interscience, New York, 1958. MR 22 \#8302.

5. H. L. Royden, Function algebras, Bull. Amer. Math. Soc. 69 (1963), 281-298. MR $26 \# 6817$.

6. J. Wermer, Analytic discs in maximal ideal spaces, Amer. J. Math. 86 (1964), 161-170. MR 28 \#5355.

University of Connecticut, Storrs, Connecticut 06268 